DF/IST-4.2001

gr-qc/0105103

\title{
QUASI-NORMAL MODES \\ OF SCHWARZSCHILD ANTI-DE SITTER BLACK HOLES: ELECTROMAGNETIC AND GRAVITATIONAL PERTURBATIONS
}

\author{
Vitor Cardoso' \\ CENTRA, Departamento de Física, Instituto Superior Técnico, \\ Av. Rovisco Pais 1, 1096 Lisboa, Portugal. \\ José P. S. Lemos] \\ CENTRA, Departamento de Física, Instituto Superior Técnico, \\ Av. Rovisco Pais 1, 1096 Lisboa, Portugal.
}

\begin{abstract}
We study the quasi-normal modes (QNM) of electromagnetic and gravitational perturbations of a Schwarzschild black hole in an asymptotically Anti-de Sitter (AdS) spacetime. Some of the electromagnetic modes do not oscillate, they only decay, since they have pure imaginary frequencies. The gravitational modes show peculiar features: the odd and even gravitational perturbations no longer have the same characteristic quasinormal frequencies. There is a special mode for odd perturbations whose behavior differs completely from the usual one in scalar and electromagnetic perturbation in an AdS spacetime, but has a similar behavior to the Schwarzschild black hole in an asymptotically flat spacetime: the imaginary part of the frequency goes as $\frac{1}{r_{+}}$, where $r_{+}$is the horizon radius. We also investigate the small black hole limit showing that the imaginary part of the frequency goes as $r_{+}^{2}$. These results are important to the AdS/CFT conjecture since according to it the QNMs describe the approach to equilibrium in the conformal field theory.
\end{abstract}

\footnotetext{
${ }^{1}$ E-mail: vcardoso@fisica.ist.utl.pt

${ }^{2}$ E-mail: lemos@kelvin.ist.utl.pt
} 


\section{Introduction}

QNMs of black holes play an important role in the study of the dynamics outside black holes. They appear, for instance, when one deals with the evolution of some field in the black hole spacetime, or in black hole-black hole collision processes. Numerical simulations ranging from the formation of a black hole in a gravitational collapse [1] to the collision of two black holes [2] provide clear evidence that no matter how one perturbs a black hole, its response will be dominated by the QNMs. QNMs allow us not only to test the stability of the event horizon against small perturbations, but also to probe the black hole mass, electric charge and angular momentum, through their characteristic waveform.

A great deal of effort has been spent to calculate the QNMs and their associated frequencies. New powerful methods, both analytical and numerical have been developed. The main interest in these studies is in the application to the analysis of the data from the gravitational waves to be detected by the

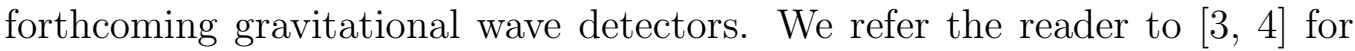
reviews. In a different context, York [5] tried to explain the thermal quantum radiance of a Schwarzschild black hole in terms of quantum zero-point fluctuations of zero mean in the QNMs.

All these previous works deal with asymptotically flat spacetimes, but the recent AdS/CFT correspondence conjecture [6] makes the investigation of QNMs in anti-de Sitter spacetimes more appealing. According to it, the black hole corresponds to a thermal state in the conformal field theory, and the decay of the test field in the black hole spacetime, corresponds to the decay of the perturbed state in the CFT. The dynamical timescale for the return to thermal equilibrium is very hard to compute directly, but can be done relatively easily using the AdS/CFT correspondence. Horowitz and Hubeny [7] (see also [8]) began the study of QNMs in AdS, by thoroughly investigating scalar perturbations in 4, 5 and 7 spacetime dimensions. Subsequently, Wang and et al [9, 10] analyzed scalar QNMs in a Reissner-Nordstöm AdS geometry. Recently, Cardoso and Lemos [11] found an exact solution for the QNMs of scalar, electromagnetic and Weyl perturbations of a BTZ black hole. Another

conjecture is related to the speculation [7, 12, 14] that there might be a connection between the critical exponent of Choptuik [13] and the imaginary part of the frequency, for small black holes. This is still an open question. 
In this paper we shall go beyond the scalar perturbations [7, 9, 10], and consider electromagnetic and gravitational perturbations of a Schwarzschild black hole in an asymptotically AdS spacetime. Electromagnetic perturbations are of interest due to the AdS/CFT conjecture since they can be seen as perturbations for some generic supergravity gauge field. In addition, the Maxwell field is an important field with different features from scalar or gravitational fields, which makes it worth studying. On the other hand, gravitational perturbations have the additional interest of arising from any other type of perturbation, be it scalar, electromagnetic, Weyl, etc., which in turn disturb the background geometry. Therefore, questions like the stability of spacetime for scalar or other perturbations, have a direct dependence on the stability to gravitational perturbations.

We will find that in the case of electromagnetic perturbations of large black holes, the characteristic QNM frequencies have only an imaginary part, and scale with the horizon radius. As for gravitational perturbations, there are two novel features. First, contrary to the asymptotically flat spacetime case, odd and even perturbations no longer have the same spectra, although in certain limits one can still prove that the frequencies are almost the same. The second intriguing result is that, for odd perturbations, there is a mode with a totally different behavior from that found in the scalar and electromagnetic case: in this mode the frequency scales with $\frac{1}{r_{+}}$, just as in asymptotically flat Schwarzschild spacetime. We also investigate the small black hole limit (a problem recently addressed by Zhu et al [15]), and find that the QNM frequencies go as $r_{+}^{2}$.

\section{Electromagnetic and Gravitational pertur- bations in a Schwarzschild AdS background}

\subsection{Maxwell perturbations}

We consider the evolution of a Maxwell field in a Schwarzschild-anti-de Sitter spacetime with metric given by

$$
d s^{2}=f(r) d t^{2}-\frac{d r^{2}}{f(r)}-r^{2}\left(d \theta^{2}+\sin ^{2} \theta d \phi^{2}\right),
$$


where, $f(r)=\left(\frac{r^{2}}{R^{2}}+1-\frac{2 M}{r}\right), R$ is the AdS radius and $M$ the black hole mass. The evolution is governed by Maxwell's equations:

$$
F_{; \nu}^{\mu \nu}=0 \quad, F_{\mu \nu}=A_{\nu, \mu}-A_{\mu, \nu},
$$

where a comma stands for ordinary derivative and a semi-colon for covariant derivative. As the background is spherically symmetric, we can expand $A_{\mu}$ in 4-dimensional vector sphericall harmonics (see [16]):

$$
A_{\mu}(t, r, \theta, \phi)=\sum_{l, m}\left(\left[\begin{array}{c}
0 \\
0 \\
\frac{a^{l m}(t, r)}{\sin \theta} \partial_{\phi} Y_{l m} \\
-a^{l m}(t, r) \sin \theta \partial_{\theta} Y_{l m}
\end{array}\right]+\left[\begin{array}{c}
f^{l m}(t, r) Y_{l m} \\
h^{l m}(t, r) Y_{l m} \\
k^{l m}(t, r) \partial_{\theta} Y_{l m} \\
k^{l m}(t, r) \partial_{\phi} Y_{l m}
\end{array}\right]\right)
$$

where the first term in the right-hand side has parity $(-1)^{l+1}$ and the second term has parity $(-1)^{l}, m$ is the azimuthal number and $l$ the angular quantum number. If we put this expansion into Maxwell's equations (2) we get a second order differential equation for the perturbation:

$$
\frac{\partial^{2} \Psi(r)}{\partial r_{*}^{2}}+\left[\omega^{2}-V(r)\right] \Psi(r)=0
$$

where the wavefunction $\Psi(r)$ is a linear combination of the functions $f^{l m}$, $h^{l m}, k^{l m}$ and $a^{l m}$ as appearing in (3). $\Psi$ has a different functional dependence according to the parity: for odd parity, i.e, $(-1)^{l+1}, \Psi$ is explicitly given by $\Psi=a^{l m}$ whereas for even parity $(-1)^{l}$ it is given by $\Psi=\frac{r^{2}}{l(l+1)}\left(-i \omega h^{l m}-\frac{d f^{l m}}{d r}\right)$, see [16] for further details. It is assumed that the time dependence is $\Psi(t, r)=e^{-i \omega t} \Psi(r)$. The potential $V$ appearing in equation (4) is given by

$$
V(r)=f(r)\left[\frac{l(l+1)}{r^{2}}\right],
$$

and the tortoise coordinate $r_{*}$ is defined as

$$
\frac{\partial r}{\partial r_{*}}=f(r) \text {. }
$$

We can of course rescale $r, r \rightarrow \frac{r}{R}$ and if we do this, the wave equation again takes the form (田) with rescaled constants i.e., $r_{+} \rightarrow \frac{r_{+}}{R}, \omega \rightarrow \omega R$, where $r_{+}$is the horizon radius. So, we can take $R=1$ and measure everything in terms of $R$. 


\section{$2.2 \quad$ Gravitational perturbations}

When dealing with first order gravitational perturbations one supposes that, at least in some restricted region of spacetime, the metric functions can be written as

$$
g_{a b}\left(x^{\nu}\right)=g_{a b}^{(0)}\left(x^{\nu}\right)+h_{a b}\left(x^{\nu}\right),
$$

where the metric $g_{a b}^{(0)}\left(x^{\nu}\right)$ is the background metric, given by some known solution of Einstein's equations, and $h_{a b}\left(x^{\nu}\right)$ is a small perturbation. Our background metric is a Schwarszchild-anti-de Sitter metric (11) and the metric $g_{a b}\left(x^{\nu}\right)$ will follow Einstein's equations in vacuum with a cosmological constant:

$$
G_{a b}-\Lambda g_{a b}=0
$$

Upon substituting (17) in (8) we will obtain some differential equations for the perturbations. We use the same perturbations as originally given by Regge and Wheeler [18], retaining their notation. After a decomposition in tensorial spherical harmonics (see Zerilli [19] and Mathews [20]), these fall into two distinct classes - odd and even - with parities $(-1)^{l+1}$ and $(-1)^{l}$ respectively, where $l$ is the angular momentum of the particular mode. While working in general relativity one has some gauge freedom in choosing the elements $h_{a b}\left(x^{\nu}\right)$ and one should take advantage of that freedom in order to simplify the rather lengthy calculations involved in computing (8). We shall therefore work with the classical Regge-Wheeler gauge in which the canonical form for the perturbations is (see also [21]):

odd parity:

$$
h_{\mu \nu}=\left[\begin{array}{cccc}
0 & 0 & 0 & h_{0}(r) \\
0 & 0 & 0 & h_{1}(r) \\
0 & 0 & 0 & 0 \\
h_{0}(r) & h_{1}(r) & 0 & h_{0}(r)
\end{array}\right] e^{-i \omega t}\left(\sin \theta \frac{\partial}{\partial \theta}\right) P_{l}(\cos \theta) ;
$$

even parity:

$h_{\mu \nu}=\left[\begin{array}{cccc}H_{0}(r) f(r) & H_{1}(r) & 0 & 0 \\ H_{1}(r) & H_{2}(r) / f(r) & 0 & 0 \\ 0 & 0 & r^{2} K(r) & 0 \\ 0 & 0 & 0 & r^{2} K(r) \sin ^{2} \theta\end{array}\right] e^{-i \omega t} P_{l}(\cos \theta) \cdot(10)$ 
Here $P_{l}(\cos \theta)$ is the Legendre polynomial with angular momentum $l$. If we put this decomposition into Einstein's equations we get ten coupled second order differential equations that fully describe the perturbations: three equations for odd perturbations and seven for even perturbations. It is however possible to circumvent the task of solving these coupled equations. Regge and Wheeler [18] and Zerilli [23] showed how to combine these ten equations into two second order differential equations, one for each parity. So following Regge and Wheeler [18] (see also [22 for more details) we define, for odd parity the wave function $Q(r)$ given by perturbations,

$$
Q(r)=\frac{f(r)}{r} h_{1}(r) .
$$

After some work, Einstein's equations yield

$$
\frac{\partial^{2} Q}{\partial r_{*}^{2}}+\left[\omega^{2}-V_{\text {odd }}(r)\right] Q=0
$$

where

$$
V_{\text {odd }}=f(r)\left[\frac{l(l+1)}{r^{2}}-\frac{6 m}{r^{3}}\right] .
$$

Likewise, following Zerilli [23] one can define for even modes the wavefunction $T(r)$ implicitly in terms of $H_{0}, H_{1}$ and $K$, through the equations

$$
\begin{array}{r}
K=\frac{6 m^{2}+c(1+c) r^{2}+m\left(3 c r-3 \frac{r^{3}}{R^{2}}\right)}{r^{2}(3 m+c r)} T+\frac{d T}{d r_{*}}, \\
H_{1}=-\frac{i \omega\left(-3 m^{2}-3 c m r+c r^{2}-3 m \frac{r^{3}}{R^{2}}\right)}{r(3 m+c r) f(r)} T-i \omega \frac{r}{f(r)} \frac{d T}{d r_{*}},
\end{array}
$$

where $c=\frac{1}{2}[l(l+1)-2]$. Then Einstein's equations for even parity perturbations can be written as

$$
\frac{\partial^{2} T}{\partial r_{*}^{2}}+\left[\omega^{2}-V_{\text {even }}(r)\right] T=0
$$

with

$$
V_{\text {even }}=\frac{2 f(r)}{r^{3}} \frac{9 m^{3}+3 c^{2} m r^{2}+c^{2}(1+c) r^{3}+3 m^{2}\left(3 c r+3 \frac{r^{3}}{R^{2}}\right)}{(3 m+c r)^{2}}
$$


Now, by defining

$$
W=\frac{2 m}{r^{2}}+\frac{-3-2 c}{3 r}+\frac{3 c^{2}+2 c^{2}+27 \frac{m^{2}}{R^{2}}}{3 c(3 m+c r)}+j,
$$

where $j=-\frac{1}{3}\left(\frac{c}{m}+\frac{c^{2}}{m}+\frac{9 m}{c R^{2}}\right)$, we obtain

$$
V_{\text {odd }}=W^{2}+\frac{d W}{d r_{*}}+\beta, \quad V_{\text {even }}=W^{2}-\frac{d W}{d r_{*}}+\beta,
$$

where $\beta=-\frac{c^{2}+2 c^{3}+c^{4}}{9 m^{2}}$. It is interesting to note that the two potentials, odd and even, can be written in such a simple form, a fact which seems to have been discovered by Chandrasekhar [25]. Potentials related in this manner are sometimes called super-partner potentials [24]). We note that similar equations were obtained by Mellor and Moss [26] for Schwarzschild-de Sitter spacetime, using a different approach.

\section{Quasinormal modes and some of its prop- erties}

\subsection{Analytical properties}

To solve equation (41) for Maxwell fields and equations (12-16) for gravitational fields, one must specify boundary conditions. Consider first the case of a Schwarzschild black hole in an asymptotically flat spacetime (see, e.g., [3]). Since in this case the potential vanishes at both infinity and horizon, the two solutions near these points are plane waves of the type $\Psi \sim e^{ \pm i \omega r_{*}}$, where the $r_{*}$ coordinate in this case ranges from $-\infty$ to $\infty$. Quasinormal modes are defined by the condition that at the horizon there are only ingoing waves, i.e., $\Psi_{\text {hor }} \sim e^{-i \omega r_{*}}$. Furthermore, one does not want to have fields coming in from infinity (where the potential in this case vanishes). So, there is only a purely outgoing wave at infinity, i.e., $\Psi_{\infty} \sim e^{i \omega r_{*}}$. Only a discrete set of complex frequencies $\omega$ meet these requirements.

Consider now a Schwarzschild black hole in an asymptotically AdS spacetime. The boundary condition at the horizon is the same, we want that near the horizon $\Psi_{\text {hor }} \sim e^{-i \omega r_{*}}$. However, $r_{*}$ has a finite range, so the second 
boundary condition needs to be modified. There have been several papers discussing which boundary conditions one should impose at infinity in AdS spacetimes ([27, 28, 29]). We shall require energy conservation and thus adopt the reflective boundary conditions at infinity 27. This means that the wavefunction is zero at infinity. For a different boundary condition see [30].

We now show that the imaginary part of the frequency $\omega$ is negative, for waves satisfying these boundary conditions, provided the potential $V$ is positive. The proof proceeds as for the scalar field perturbation case [0], although there are some steps we think are useful to display explicitly here. Writing $\phi$ for a generic wavefunction as

$$
\phi=e^{i \omega r_{*}} Z,
$$

where, $Z$ can be $\Psi, Q$ or $T$, we find

$$
f(r) \frac{\partial^{2} \phi}{\partial r^{2}}+\left[f^{\prime}-2 i \omega\right] \frac{\partial \phi}{\partial r}-\frac{V}{f} \phi=0,
$$

where $f=\left(r^{2}+1-\frac{2 M}{r}\right)$. In the proof, we are going to need the asymptotic behavior of the solutions of equation (21). For $r \rightarrow r_{+}$we have $f \sim\left(3 r_{+}+\frac{1}{r_{+}}\right)\left(r-r_{+}\right)$and $\frac{V}{f} \sim C$, where $\mathrm{C}$ is a constant which takes different values depending on the case, electromagnetic, odd or even gravitational perturbations. So equation (21) becomes, in this limit,

$$
A y \frac{\partial^{2} \phi}{\partial y^{2}}+[A-2 i \omega] \frac{\partial \phi}{\partial y}-C \phi=0
$$

where $y=r-r_{+}$, and $A=3 r_{+}+\frac{1}{r_{+}}$. This equation has an exact solution in terms of the modified Bessel functions $I_{\nu}(z)$ [31,

$$
\phi=C_{1} y^{i \frac{\omega}{A}} I_{-\frac{i \omega}{A}}\left(2\left(\frac{C}{A} y\right)^{\frac{1}{2}}\right)+C_{2} y^{i \frac{\omega}{A}} I_{\frac{i \omega}{A}}\left(2\left(\frac{C}{A} y\right)^{\frac{1}{2}}\right) .
$$

We want the asymptotic behavior of these functions when $y \rightarrow 0$ which is given by $I_{\nu}(z) \rightarrow \frac{\left(\frac{z}{2}\right)^{\nu}}{\Gamma(\nu+1)}, z \rightarrow 0$. So, near the horizon the wavefunction $\phi$ behaves as

$$
\phi_{r_{+}}=C_{1} \frac{\left(\frac{C}{A}\right)^{-\frac{i \omega}{A}}}{\Gamma\left(1-\frac{2 i \omega}{A}\right)}+C_{2} \frac{y^{\frac{2 i \omega}{A}}\left(\frac{C}{A}\right)^{\frac{i \omega}{A}}}{\Gamma\left(1+\frac{2 i \omega}{A}\right)} .
$$


We can see that if one wants to rule out outgoing modes at the horizon, we must have $C_{2}=0$, so that $\phi$ in equation (20) does not depend on $y$. Let's now investigate the asymptotic behavior at infinity. For $r \rightarrow \infty$ we have $\frac{V}{f} \rightarrow \frac{l(l+1)}{r^{2}}$. Therefore near infinity equation (21) becomes

$$
r^{2} \frac{\partial^{2} \phi}{\partial r^{2}}+[2 r-2 i \omega] \frac{\partial \phi}{\partial r}-\frac{l(l+1)}{r^{2}} \phi=0 .
$$

Putting $x=\frac{1}{r}$ we have

$$
\frac{\partial^{2} \phi}{\partial x^{2}}+2 i \omega \frac{\partial \phi}{\partial x}-l(l+1) \phi=0
$$

with solution $\phi=\phi_{\infty}$ given by

$$
\phi_{\infty}(x)=A \mathrm{e}^{\left[-i \omega+i\left(\omega^{2}-l(l+1)\right)^{1 / 2}\right] x}+B \mathrm{e}^{\left[-i \omega-i\left(\omega^{2}-l(l+1)\right)^{1 / 2}\right] x}
$$

Now, $\phi_{\infty}(x=0)=0$, therefore $A=-B$, and thus,

$$
\phi_{\infty}(x)=A \mathrm{e}^{-i \omega x} \sin \left[\left(\omega^{2}-l(l+1)\right)^{\frac{1}{2}} x\right]
$$

We can now proceed in the proof. Multiplying equation 21) by $\bar{\phi}$ (the complex conjugate of $\phi$ ), and integrating from $r_{+}$to $\infty$ we obtain

$$
\int_{r_{+}}^{\infty} d r\left[\bar{\phi} \frac{d\left(f \frac{d \phi}{d r}\right)}{d r}-2 i \omega \bar{\phi} \frac{d \phi}{d r}-\frac{V}{f} \bar{\phi} \phi\right]=0 .
$$

Integrating by parts yields

$$
\int_{r_{+}}^{\infty} d r\left[\frac{d\left[\bar{\phi} f \frac{d \phi}{d r}\right]}{d r}-f\left|\frac{d \phi}{d r}\right|^{2}-2 i \omega \bar{\phi} \frac{d \phi}{d r}-\frac{V}{f}|\phi|^{2}\right]=0 .
$$

Now, one can show that $\left[\bar{\phi} f \frac{d \phi}{d r}\right]_{r_{+}}=0$, in order to satisfy the boundary conditions. Indeed, at $r_{+}, \phi\left(r_{+}\right)=$constant and $f\left(r_{+}\right)=0$. Now, at infinity, even though $\bar{\phi}(\infty)=0$, we have also $f(\infty)=\infty$, so we have to show that $\left[\bar{\phi} f \frac{d \phi}{d r}\right]_{\infty}=0$. From equation (28) we can check that this is indeed true. Thus, equation $(30)$ gives

$$
\int_{r_{+}}^{\infty} d r\left[f\left|\frac{d \phi}{d r}\right|^{2}+2 i \omega \bar{\phi} \frac{d \phi}{d r}+\frac{V}{f}|\phi|^{2}\right]=0 .
$$


Taking the imaginary part of (31) we have

$$
\int_{r_{+}}^{\infty} d r\left[\omega \bar{\phi} \frac{d \phi}{d r}+\bar{\omega} \phi \frac{d \bar{\phi}}{d r}\right]=0
$$

wich, after an integration by parts reduces to

$$
(\omega-\bar{\omega}) \int_{r+}^{\infty} d r\left[\bar{\phi} \frac{d \phi}{d r}\right]=\bar{\omega}\left|\phi\left(r_{+}\right)\right|^{2} .
$$

Finally, inserting this back into (31) yields

$$
\int_{r_{+}}^{\infty} d r\left[f\left|\frac{d \phi}{d r}\right|^{2}+\frac{V}{f}|\phi|^{2}\right]=-\frac{|\omega|^{2}\left|\phi\left(r_{+}\right)\right|^{2}}{\operatorname{Im} \omega} .
$$

From this relation, one can infer that, if $\mathrm{V}$ is positive definite then $\operatorname{Im} \omega<0$ necessarily. So, since electromagnetic and even gravitational perturbations have $V>0$ one always has $\operatorname{Im} \omega<0$. As for odd gravitational perturbations there are instances where $V<0$, making this theorem unreliable for these

cases. However, for $r_{+}<\left[\frac{l(l+1)}{3}-1\right]^{\frac{1}{2}}$, i.e., small enough masses, $V>0$ (see equation $(\sqrt{13})$ ), and the theorem applies.

Another important point concerns the late time behavior of these fields, and the existence or not of power-law tails. As shown by Ching et al [32], for potentials that vanish exponentially near the horizon, there are no power-law tails, so there will be no such tails in our case.

\subsection{Numerical Calculation of the QNM frequencies}

To find the frequencies $\omega$ that satisfy the boundary conditions we first note that equation (21) has only regular singularities in the range of interest. It has therefore, by Fuchs theorem, a polynomial solution [33]. To deal with the point at infinity, we first change the independent variable $r$ to $x=\frac{1}{r}$. Now we can use Fröbenius method by looking for an indicial equation (for further details see [7]), and force it to obey the boundary condition at the horizon $\left(x=\frac{1}{r_{+}}=h\right)$. We get the following solution to equation (21),

$$
\phi(x)=\sum_{n=0}^{\infty} a_{n(\omega)}(x-h)^{n}
$$


where $a_{n(\omega)}$ is a function of the frequency. If we put (35) into (21) and use the boundary condition $\phi=0$ at infinity $(x=0)$ we obtain

$$
\sum_{n=0}^{\infty} a_{n(\omega)}(-h)^{n}=0 .
$$

Our problem is reduced to that of finding a numerical solution of the polynomial equation (36). The numerical roots for $\omega$ of equation (36) can be evaluated resorting to numerical computation. Obviously, one cannot determine the full sum in expression (36), so we have to determine a partial sum from 0 to $N$, say and find the roots $\omega$ of the resulting polynomial expression. We then move onto the next term $N+1$ and determine the roots. If the method is reliable, the roots should converge. We stop our search when we have a 3 decimal digit precision.

\subsubsection{Electromagnetic modes}

As long as the modes are decaying, it does not matter whether they're oscillating or not. However, as we will see there are frequencies in the electromagnetic case with a vanishing real part, which makes it possible to use an approximation, due to Liu [17], to the highly damped modes. Although the method was originally developed for the asymptotically flat space, it is quite straightforward to apply it to our case. There is therefore a way to test our results. Unfortunately, this method relies heavily on having a frequency with a large pure imaginary part, so as we shall see it will only work for electromagnetic perturbations. We have computed the lowest frequencies for some values of the horizon radius $r_{+}$, and $l$. The frequency is written as $\omega=\omega_{r}+i \omega_{i}$, where $\omega_{r}$ is the real part of the frequency and $\omega_{i}$ is its imaginary part. In tables 1 and 2 we list the numerical values of the lowest quasinormal frequencies of electromagnetic perturbations for $l=1$ and $l=2$ and for selected values of $r_{+}$. For frequencies with no real part, we list the values obtained in Liu's aproximation. 


\begin{tabular}{|l|l|l|l|l|}
\hline & \multicolumn{2}{|c|}{ Numerical } & \multicolumn{2}{l|}{ Liu's approximation } \\
\hline$r_{+}$ & $-\omega_{i}$ & $\omega_{r}$ & $-\omega_{i}$ & $\omega_{r}$ \\
\hline 0.8 & 1.287 & 2.175 & - & - \\
\hline 1 & 1.699 & 2.163 & - & - \\
\hline 5 & 8.795 & $\sim 0$ & 7.6 & $\sim 0$ \\
\hline 10 & 15.506 & $\sim 0$ & 15.05 & $\sim 0$ \\
\hline 50 & 75.096 & $\sim 0$ & 75.01 & $\sim 0$ \\
\hline 100 & 150.048 & $\sim 0$ & 150.005 & $\sim 0$ \\
\hline
\end{tabular}

Table 1. Lowest QNM of electromagnetic perturbations for $l=1$. The - in Liu's approximation columns means that the method is not applicable.

\begin{tabular}{|l|l|l|l|l|}
\hline & \multicolumn{2}{|c|}{ Numerical } & \multicolumn{2}{l|}{ Liu's approximation } \\
\hline$r_{+}$ & $-\omega_{i}$ & $\omega_{r}$ & $-\omega_{i}$ & $\omega_{r}$ \\
\hline 0.8 & 1.176 & 2.501 & - & - \\
\hline 1 & 1.579 & 2.496 & - & - \\
\hline 5 & 10.309 & 0.822 & 7.6 & $\sim 0$ \\
\hline 10 & 15.755 & $\sim 0$ & 15.05 & $\sim 0$ \\
\hline 50 & 75.139 & $\sim 0$ & 75.01 & $\sim 0$ \\
\hline 100 & 150.069 & $\sim 0$ & 150.005 & $\sim 0$ \\
\hline
\end{tabular}

Table 2. Lowest QNM of electromagnetic perturbations for $l=2$.

As one can see, the imaginary part of the frequency, which determines how damped the mode is, and which according to the AdS/CFT conjecture is a measure of the characteristic time $\tau=\frac{1}{\omega_{i}}$ of approach to thermal equilibrium, scales linearly (for large black holes) with the horizon radius supporting the arguments given in [7]. Moreover, the frequencies do not seem to depend on the angular quantum number $l$, and are in excellent agreement with the analytical approximation for strongly damped modes.

For a better visualization we also plot $\omega_{i} \times r_{+}$in Figure 1 . 


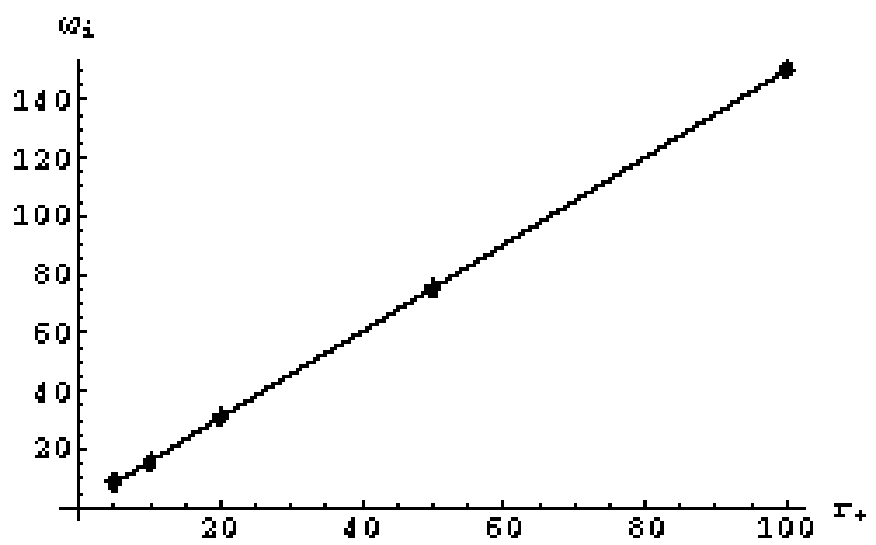

Figure 1. Lowest electromagnetic QNM for $l=1$ as function of $r+$. The black lozenges represent some frequencies numerically calculated. The line connecting them is a linear fit.

\subsubsection{Gravitational modes}

The numerical calculation of the quasinormal frequencies for gravitational perturbations proceeds as outlined previously (the associated differential equation has only regular singularities, so it is possible to use an expansion such as (35)).

(i) Odd modes: In tables 3 and 4 we show the two lowest QNM frequencies for $l=2$ and $l=3$ odd gravitational perturbations. An important point in odd QNMs is that there is a mode for which the frequencies are not only pure imaginary and very small, but also scale with $\frac{1}{r_{+}}$! This is similar to the behavior of Schwarzschild black holes in asymptotically flat spacetimes, as mentioned. However, all frequencies have a negative imaginary part, which indicates that the spacetime is stable for this kind of perturbations. 


\begin{tabular}{|l|l|l|l|l|}
\hline & \multicolumn{2}{|l|}{ lowest QNM } & \multicolumn{2}{l|}{ second lowest QNM } \\
\hline$r_{+}$ & $-\omega_{i}$ & $\omega_{r}$ & $-\omega_{i}$ & $\omega_{r}$ \\
\hline 0.5 & 6.4 & 0 & 0.72 & 3.037 \\
\hline 1 & $\sim 2(?)$ & 0 & 2.404 & 3.033 \\
\hline 2 & 0.728 & $\sim 0$ & 5.258 & 4.447 \\
\hline 5 & 0.2703 & $\sim 0$ & 13.294 & 9.577 \\
\hline 10 & 0.13378 & $\sim 0$ & 26.626 & 18.662 \\
\hline 50 & 0.02667 & $\sim 0$ & 133.19 & 92.505 \\
\hline 100 & 0.0132 & $\sim 0$ & 266.384 & 184.959 \\
\hline
\end{tabular}

Table 3. Lowest QNM of gravitational odd perturbations for $l=2$.

\begin{tabular}{|l|l|l|l|l|}
\hline & \multicolumn{2}{|l|}{ lowest QNM } & \multicolumn{2}{l|}{ second lowest QNM } \\
\hline$r_{+}$ & $-\omega_{i}$ & $\omega_{r}$ & $-\omega_{i}$ & $\omega_{r}$ \\
\hline 1 & 10 & 0 & 1.639 & 3.849 \\
\hline 2 & 2.189 & 0 & 5.080 & 4.615 \\
\hline 5 & 0.690 & $\sim 0$ & 13.247 & 9.735 \\
\hline 10 & 0.336 & $\sim 0$ & 26.603 & 18.742 \\
\hline 50 & 0.0669 & $\sim 0$ & 133.19 & 92.521 \\
\hline 100 & 0.0333 & $\sim 0$ & 266.382 & 184.967 \\
\hline
\end{tabular}

Table 4. Lowest QNM of gravitational odd perturbations for $l=3$.

The value $\omega=2$ in Table 3 marked with a "?" is a somewhat dubious result. In fact, from (24) it follows that if $1-\frac{2 i \omega}{A}=-n$, with $n$ an integer, then there is nothing going down the hole, so perhaps it is not a QNM. It is also an "algebraically special value" in the sense of Chandrasekhar [34].

(ii) Even modes: In table 5 we show the lowest QNM frequencies for $l=2$ and $l=3$ even gravitational perturbations. We point out the remarkable resemblance of the values in table 3 with those in [7] for scalar perturbations, even though the potentials are quite different. We have performed calculations for higher values of the angular quantum number $l$, and found that the QNM frequencies are indeed very similar throughout all values of $l$. 


\begin{tabular}{|l|l|l|l|l|}
\hline & \multicolumn{2}{|l|}{ lowest QNM, $l=2$} & \multicolumn{2}{l|}{ lowest QNM, $l=3$} \\
\hline$r_{+}$ & $-\omega_{i}$ & $\omega_{r}$ & $-\omega_{i}$ & $\omega_{r}$ \\
\hline 1 & 1.584 & 3.018 & 1.392 & 3.909 \\
\hline 2 & 3.974 & 4.546 & 3.299 & 4.597 \\
\hline 5 & 12.649 & 9.83 & 11.642 & 10.217 \\
\hline 10 & 26.301 & 18.806 & 25.788 & 19.089 \\
\hline 50 & 133.125 & 92.535 & 133.022 & 92.596 \\
\hline 100 & 266.351 & 184.959 & 266.300 & 185.005 \\
\hline
\end{tabular}

Table 5. Lowest QNM of gravitational even perturbations.

(iii) Discussion: We first note that there is clearly a distinction between odd and even perturbations: they no longer have the same spectra, contrary to the asymptotically flat space case (see [35]), a problem we shall consider in more detail in the next subsection. We also remark that in electromagnetic and scalar perturbations the frequency scales with $r_{+}$(for large black holes at least). Since the temperature scales also with $r_{+}$in the large black hole regime, this means that the frequency scales with the temperature. Thus, in the dual CFT the approach to thermal equilibrium is faster for higher temperatures. This is a totally different behavior from that of asymptotically flat space, in which the frequency scales with $\frac{1}{r_{+}}$. However, for odd modes there is one that scales with $\frac{1}{r_{+}}$. This is a reflection of the different behavior of the potential $V_{\text {odd }}$ for odd perturbations (that was why we couldn't prove stability for odd perturbations in the first place), and of the boundary conditions, as we shall show in section 3.3. The odd modes are therefore particularly long-lived.

\subsection{On the isospectrality breaking between odd and even perturbations}

As is well known 25, 35] in the case of a Schwarzschild black hole in an asymptotically flat space the two potentials $V_{\text {even }}$ and $V_{\text {odd }}$ give rise to the same quasinormal frequencies (in fact to the same absolute value of the reflection and transmission coefficients). This remarkable property followed from a special relation (the equivalent for asymptotically flat spacetimes of our 
equation (19) ) between the potentials and the behavior of $\mathrm{W}$ at the boundaries. However, as one can see in tables 3,4 and 5 there is a isospectrality breaking between odd and even perturbations in Schwarzschild anti-de Sitter spacetime.

We shall now treat this problem. The breaking of the isospectrality is intimately related to the behavior of $W$ at infinity. On taking advantage of the machinery developed by Chandrasekhar, we seek a relation between odd and even perturbations of the form

$$
\begin{aligned}
& Q=p_{1} T+q_{1} \frac{d T}{d r_{*}}, \\
& T=p_{2} Q+q_{2} \frac{d Q}{d r_{*}},
\end{aligned}
$$

yielding (see 25] for details), $q_{1}^{2}=\frac{1}{\beta-\omega^{2}}, p_{1}=q W, p_{2}=-p_{1}$ and $q_{2}=q_{1}=$ $q$. Thus, we obtain

$$
\begin{gathered}
Q=q W T+q \frac{d T}{d r_{*}}, \\
T=-q W Q+q \frac{d Q}{d r_{*}} .
\end{gathered}
$$

Suppose now that $\omega$ is a QNM frequency of $T$, i.e., one for which

$$
\begin{array}{r}
T \rightarrow A_{\mathrm{even}} e^{-i \omega r_{*}}, r \rightarrow r_{+}, \\
T \rightarrow 0, r \rightarrow \infty
\end{array}
$$

Substituting this into equation (40) we see that

$$
\begin{aligned}
& Q \rightarrow A_{\mathrm{even}} q[W(r+)-i \omega] e^{-i \omega r_{*}}, r \rightarrow r_{+}, \\
& Q \rightarrow q\left(\frac{d T}{d r_{*}}\right)_{r=\infty}, r \rightarrow \infty .
\end{aligned}
$$

However, from equation $(28),\left(\frac{d T}{d r_{*}}\right)_{r=\infty}$ is in general not zero so that $\omega$ will in general fail to be a QNM frequency for Q. Should $Q$ and $T$ be smooth functions of $\omega$, one expects that if $q$ is "almost zero" then $\omega$ should "almost" be a QNM frequency for $Q$. Now, the condition that $q$ is almost zero is that $\beta-\omega^{2}$ be very large, and one expects this to be true either when $\omega$ 
is very large or else when $\beta$ is very large. And in fact, as one can see in tables 3, 4 and 5 for very large $\omega$ the frequencies are indeed almost identical. On the other hand, for very small black holes ( $\beta$ very large) one expects the frequencies to be exactly the same, since both potentials have the same asymptotic behavior in this regime, as we shall see in the next section. One would be tempted to account for the remarkable resemblances between QNM frequencies of scalar and gravitational perturbations by a similar approach, but the proof is still eluding us. Should such an approach work, it could be of great importance not only to this specific problem, but also to the more general problem of finding the asymptotic distribution of eigenvalues, by studying a different potential with (asymptotically) the same eigenvalues, but more easy to handle.

\section{The limit $\mathrm{m} \rightarrow 0$}

Although it is not possible to solve exactly for the QNM frequencies, it is possible to gain some analytical insight in the special case of very small black holes. There has been some discussion about this regime (see [15], and also [07, 36] and references therein). Here we shall exploit the behavior of QNM frequencies in this regime a little further. For very small black holes one can easily see that both potentials (electromagnetic and gravitational) look like, in the $r_{*}$ coordinate, a barrier with unequal heights:

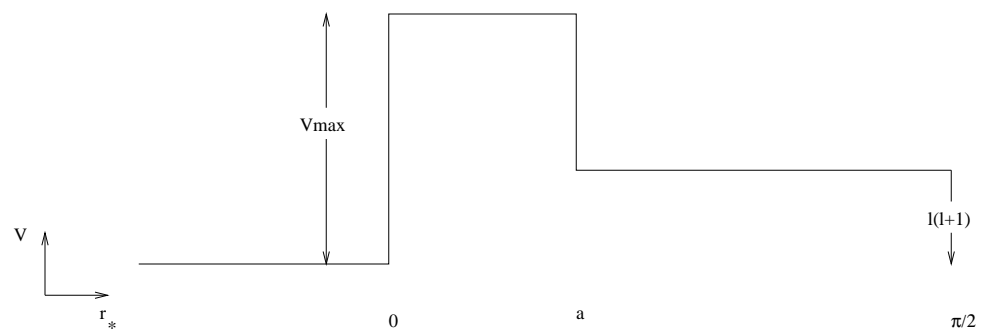

Figure 2. The potential for small black holes. $V_{\max }=\frac{4 l(l+1)}{27 r_{+}^{2}}$ and $a \sim 3 r_{+}$.

It is trivial to obtain equations for the quasinormal frequencies in this limit. If $\Psi$ is a general wavefunction then

$$
\frac{d \Psi / d r_{*}}{\Psi}=-i \omega, \quad r_{*}<0
$$




$$
\begin{gathered}
\frac{d \Psi / d r_{*}}{\Psi}=\frac{i k_{1} B e^{i k_{1} r_{*}}-i k_{1} C e^{-i k_{1} r_{*}}}{B e^{i k_{1} r_{*}}+C e^{-i k_{1} r_{*}}}, \quad 0<r_{*}<a, \\
\frac{d \Psi / d r_{*}}{\Psi}=\frac{i k D e^{i k r_{*}}-i k_{1} E e^{-i k r_{*}}}{D e^{i k r_{*}}+E e^{-i k r_{*}}}, \quad r_{*}>a,
\end{gathered}
$$

where $k_{1}=\left(\omega^{2}-V_{\max }\right)^{1 / 2}$, and $k=\left[\omega^{2}-l(l+1)\right]^{1 / 2}$. Imposing the continuity of the logarithmic derivative and furthermore that $\Psi=0$ at infinity $\left(r_{*}=\frac{\pi}{2}\right)$, we get

$$
k_{1}\left[\frac{\frac{k_{1}-\omega}{k_{1}+\omega} e^{2 i k_{1} a}-1}{\frac{k_{1}-\omega}{k_{1}+\omega} e^{2 i k_{1} a}+1}\right]=k\left[\frac{e^{2 i k\left(\frac{\pi}{2}-a\right)}+1}{1-e^{2 i k\left(\frac{\pi}{2}-a\right)}}\right] .
$$

In the limit $a \rightarrow 0, k_{1} \rightarrow \infty(m \rightarrow 0)$ we have, supposing that $\omega$ stays small, the condition $e^{2 i k \frac{\pi}{2}}=1$ which means that

$$
\omega_{0}^{2}=4 n^{2}+l(l+1), \quad n=1,2, \ldots,
$$

corresponding to a bound state. This gives for the lowest QNM frequencies $(n=1): \omega_{0}=2.45$ for $l=1$ and $\omega_{0}=3.16$ for $l=2$. The above are to be compared with those in Tables 1-5. The agreement seems excellent, and we can now go a step further : If we linearize (48) around the solution (49), i.e, if we write $\omega=\omega_{0}+i \delta$ and substitute back in (48) we obtain, to third order in $\delta$, the values listed in Table 6 .

\begin{tabular}{|c|c|c|}
\hline & $a=3 / h$ & $a=6 / h$ \\
\hline $\mathrm{l}$ & $\delta$ & $\delta$ \\
\hline 1 & $-2.1 / h-i\left(1.42 / h^{2}\right)$ & $-1.92 / h-i\left(0.05 / h^{2}\right)$ \\
\hline 2 & $-0.859 / h-i\left(0.04 / h^{2}\right)$ & $-0.85 / h-i\left(1.4 \times 10^{-4} / h^{2}\right)$ \\
\hline
\end{tabular}

Table 6 . The linearized frequency $\delta$ for selected values of the angular quantum number $l$ and the potential width $a$.

We have chosen a typical value of $a \sim \frac{3}{h}$, but we can see that, although the real part does not depend very much on $a$, the imaginary part is strongly sensitive to $a$. Nevertheless, one can be sure that whatever value of $a$, the imaginary part goes as $\frac{1}{h^{2}}$ and is always negative. 


\section{Conclusions}

We have computed the electromagnetic and gravitational QNM frequencies of Schwarzschild-AdS black holes in four dimensions. These modes dictate the late time behavior of a minimally coupled electromagnetic field and of small gravitational perturbations, respectively. The conclusions are: (i) The frequencies all have a negative imaginary part, which means that the black hole is stable against these perturbations, since these will decay exponentially with time; (ii) Maxwell perturbations are strongly damped, so according to the AdS/CFT conjecture, any electromagnetic perturbed thermal state will rapidly approach equilibrium; (iii) for odd gravitational perturbations in the large black hole regime, the imaginary part of the frequency (decaying mode) goes to zero scaling with $\frac{1}{r_{+}}$, just as in asymptotically flat space. In terms of the AdS/CFT correspondence, this implies that the greater the mass, the more time it takes to approach equilibrium, an unusual result; (iv) scalar [7] and gravitational even perturbations exhibit an amazing similarity for the characteristic time damping of the perturbations, but we have not been able to prove it analytically; (v) in the small black hole regime the imaginary part of the frequency (decaying mode) scales with $r_{+}^{2}$.

\section{Acknowledgments}

This work was partially funded by Fundação para a Ciência e Tecnologia (FCT) through project PESO/PRO/2000/4014. V.C. also acknowledges finantial support from FCT through PRAXIS XXI programme. J. P. S. L. thanks Observatório Nacional do Rio de Janeiro for hospitality. 


\section{References}

[1] C. T. Cunningham, R. H. Price, and V. Moncrief, Ap. J. Lett. 224, 643(1978).

[2] P. Anninos, D. Hobill, E. Seidel, L. Smarr and W-M. Suen, Phys. Rev. Lett. 71, 2851(1993).

[3] K. D. Kokkotas, and B. G. Schmidt, Living Rev. Rel. 2, 2(1999).

[4] N. Andersson, in Black Holes, Gravitational radiation and the Universe, (Edited by Bala R. Iyer and Biplab Bhawall, 1999).

[5] J. W. York, Phys. Rev. D28, 2929(1983).

[6] J. Maldacena, Adv. Theor. Math. Phys. 2, 253(1998).

[7] G. T. Horowitz, and V. Hubeny, Phys. Rev. D62, 024027(2000).

[8] G. T. Horowitz, Class. Quantum Grav. D17, 1107(2000).

[9] B. Wang, C. Y. Lin, and E. Abdalla, Phys. Lett. B481, 79(2000).

[10] B. Wang, C. M. Mendes, and E. Abdalla, Phys. Rev. D63, 084001(2001).

[11] V. Cardoso, J. P. S. Lemos, Phys. Rev. D63, 124015(2001), grqc/0101052.

[12] D. Birmingham, hep-th/0101194.

[13] M. W. Choptuik, Phys. Rev. Lett. 70, 9(1993).

[14] W. T. Kim, J. J. Oh, hep-th/0105103

[15] J. Zhu, B. Wang, and E. Abdalla, Phys. Rev. D63, 124004(2001).

[16] R. Ruffini, in Black Holes: les Astres Occlus, (Gordon and Breach Science Publishers, 1973).

[17] H. Liu, Class. Quantum Grav. 12, 543(1995).

[18] T. Regge, J. A. Wheeler, Phys. Rev. 108, 1063(1957). 
[19] F. Zerilli, J. Math. Phys. 11, 2203(1970).

[20] J. Mathews, J. Soc. Ind. Appl. Math. 10, 768(1962).

[21] C. V. Vishveshwara, Phys. Rev. D1, 2870(1970).

[22] L. A. Edelstein, and C. V. Vishveshwara, Phys. Rev. D1, 3514(1970).

[23] F. Zerilli, Phys. Rev. Lett. 24, 737(1970).

[24] F. Cooper, A. Khare and U. Sukhatme, Phys. Rep. 251, 267(1995).

[25] S. Chandrasekhar, in The Mathematical Theory of Black Holes, (Oxford University Press, New York, 1983).

[26] F. Mellor, I. Moss, Phys. Rev. D41, 403(1990).

[27] S. J. Avis, C. J. Isham and D. Storey, Phys. Rev. D18, 3565(1978).

[28] P. Breitenlohner and D. Z. Freedman, Phys. Lett. B115, 197(1982).

[29] C. P. Burgess and C. A. Lutken, Phys. Lett. B153, 137(1985).

[30] A. Dasgupta, Phys. Lett. B445, 279(1999).

[31] M. Abramowitz, and I. A. Stegun in Handbook of Mathematical Functions, (Dover, New York, 1970).

[32] E. S. C. Ching, P. T. Leung, W. M. Suen, and K. Young, Phys. Rev. D52, 2118(1995).

[33] G. B. Arfken, and H. J. Weber, Mathematical Methods for Physicists, (Academic Press, 1995).

[34] S. Chandrasekhar, Proc. R. Soc. London, Ser. A 392, 1(1984).

[35] S. Chandrasekhar, and S. Detweiler, Proc. R. Soc. London, Ser. A 344, 441(1975).

[36] V. Hubeny, L. Susskind, and N. Toumbas, hep-th/0011164. 